L'article du Professeur Meyer, juge expérimenté au Tribunal fédéral et auteur émérite, n'obtiendra pas forcément le plein accord de tous les lecteurs et sa légère ironie suscitera certainement le débat. Pour ma part, je trouve que son article est différencié, intelligent et compréhensible, I'un des plus beaux compliments que I'on puisse faire à un juriste rédacteur d'expertises.

Cela ne veut pas dire que tous mes confrères partageront entièrement mon avis. Cela vaut principalement pour celles et ceux qui, d'habitude, ne se confrontent guère aux points de vue différents de la médecine et du droit et aux rôles divergents du médecin traitant et de l'expert.
La proposition de l'auteur, au terme de son article, d'opter pour une indemnisation plutôt qu'une rente partagera certainement les esprits et c'est sûrement son intention. Quant à moi, je trouve cette proposition digne de considération. L'un de mes anciens professeurs à la clinique de rééducation de Bellikon l'aurait certainement saluée, lui qui estimait judicieuse et réalisable la solution d'une indemnité, notamment pour faciliter la réinsertion professionnelle.

Dr Gerhard Ebner, $M H A$, directeur des cliniques psychiatriques universitaires de Bâle

\title{
La maladie en tant que notion du droit de l'assurance sociale déterminant une prestation*
}

\section{Ulrich Meyer}

Traduction: lic. iur. et lic. oec. Françoise Bruderer, Berne

\footnotetext{
* En collaboration avec Dr. Myriam Schwendener, Secrétaire juridique auprès du Service cantonal de l'aide aux victimes, Direction de la justice et de l'intérieur du canton de Zurich.

Version raccourcie d'une conférence donnée le 24 avril 2008 lors de la 8 journée centrale à Lucerne. Pour plus de détails: Thomas Gächter/ Myriam Schwendener (édit.), Rechtsfragen zum Krankheitsbegriff, Entwicklungen in der Praxis, Tagungsband zum 8. Zentrumstag des Luzerner Zentrums für Sozialversicherungsrecht (LuZeSo).

Zurich: Schulthess; 2009. p. 5-24.
}

1 Dürrenmatt F. Der Richter und sein Henker (le juge et son bourreau). Zurich: Diogenes; 1985.

2 Myriam Schwendener, Krankheit und Recht, der Krankheitsbegriff im schweizerischen Sozialversicherungsrecht, Bâle 2008 .
La maladie, risque social de premier rang, est l'objet, dans le droit de l'assurance sociale, de la définition légale de l'art. 3 de la loi fédérale sur la partie générale du droit des assurances sociales (LPGA). Il s'agit d'une tautologie légale que le droit ne pouvait concrétiser qu'avec l'appui de la médecine. Ce que celle-ci considérait comme maladie a valu sans autre pendant des décennies dans le domaine du droit. Cette équivalence apparaît aujourd'hui de plus en plus problématique car la notion médicale de maladie a perdu ses contours par l'incorporation du comportement subjectif face à la maladie et des facteurs sociaux. Une délimitation convaincante nécessite un débat interdisciplinaire constant entre la médecine et le droit. Il convient dans ce contexte de tenir compte du fait que la notion de maladie a dans ces deux sciences pratiques une fonction tout à fait différente: en médecine la notion de maladie sert en premier lieu à estimer les risques liés à la santé et à décider ce qui doit être traité. En droit la notion de maladie sert principalement à déterminer et délimiter les prestations exigibles. Ces buts très différents expliquent en grande partie pourquoi les médecins traitants d'une part, et d'autre part, les experts médicaux et les personnes appliquant le droit, évaluent de manière souvent divergente l'incapacité de travailler d'une seule et même personne.

«Plus qu'un an répondit Hungertobel, s'assit sur une chaise accolée au mur de son cabinet et, désarmé, regarda Bärlach qui se tenait, immobile et humble, debout au milieu de la chambre, plongé dans une solitude lointaine, froide, dont le regard perdu fit à présent baisser les yeux du médecin.» [1]

\section{Remarque liminaire}

Peu d'œuvres de la littérature mondiale ne donnent un rôle, parfois déterminant, à la maladie. Nourri de ses expériences de lecture et du vécu, chacun sait, ou croit savoir, ce qu'est la maladie, la santé. Des doutes s'élèvent quand la maladie et la santé se côtoient ou se confondent. Précisément dans ces cas limites la réglementation légale assez simple de l'art. 3 LPGA nous laisse plus ou moins perplexe. Qui ose en disserter, comme le montre une dissertation zurichoise admise summa cum laude [2], concédera bien vite: sous son angle juridique, le thème livre bien peu. En effet, la notion de maladie, de par sa nature, passionne et nous défie si elle est abordée par le côté médical, notamment en considérant son développement des dernières vingt à trente années. La pratique est, comme il s'agira de le démontrer, plus importante que la réglementation légale. Pour cette raison, le thème de l'application du droit reste difficile à aborder car la question revient sans cesse de savoir comment s'y prendre avec la réglementation légale, peu pertinente en elle-même, dans un contexte médical devenu complexe et controversé.

\section{Le caractère tautologique de la définition légale}

\section{Le cœur de la notion}

L'art. 3 al. 1 LPGA s'exprime ainsi sur la notion légale de la maladie: Est réputée maladie toute atteinte à la santé physique, mentale ou psychique qui n'est pas due à un accident et qui exige un examen ou un traitement médical ou provoque une incapacité de travailler. 
3 Dont les mesures servant au diagnostic ou au traitement d'une maladie et de ses séquelles (art. 25 al. 1 LAMal). Le but du traitement médical est premièrement de retrouver ou d'améliorer la santé ou de prévenir des dangers concrets pour la vie. Le simple combat de symptômes peut également être un traitement de la maladie comme par exemple l'allègement des douleurs (des mourants). Les prestations diagnostiques, thérapeutiques et de soins sont remboursées selon l'art. 25 al. 2 let. a LAMal par

l'assurance-maladie pour autant qu'un médecin, un chiropraticien ou une personne sur pre-scription ou mandat médical effectue le traitement. Sur tout le sujet voir Gebhard Eugster, Krankenversicherung, dans: Ulrich Meyer (édit.), Soziale Sicherheit, (Schweizerisches Bundesverwaltungsrecht, tome XIV, édité par Heinrich Koller/ Georg Müller/René Rhinow/Ulrich Zimmerli), 2ème édition, Bâle/ Genève/Munich 2007, p. 337-823, nos 315 ss., 322 ss. avec indications, 328 ss. avec indications; Thomas Gächter/Irène Vollenweider, Gesundheitsrecht, ein Kurzlehrbuch, Bâle 2008, nos 712 ss.; Alfred Maurer, Das neue Krankenversicherungsrecht, Bâle/Francfort-sur-leMain 1996, p. 44 ss.

4 Eugster (note 3), nos 248 ss.

5 Ulrich Meyer-Blaser, Der Rechtsbegriff der Arbeitsunfähigkeit und seine Bedeutung in der Sozialversicherung, namentlich für den Einkommensvergleich in der Invaliditätsbemessung, dans: René Schaffhauser/Franz Schlauri (édit), Schmerz und Arbeitsunfähigkeit, tome 23 de la série IRP/HSG, St. Gall 2003, p. 27-119, 31 ss.

6 Toujours encore à conseiller et dans ses principes juste: Constantin Schuler, Medizin im Dienste des Rechts: Ärztliche Dienste von eidgenössischen Sozialversicherungen, dans: Sozialversicherungsrecht im Wandel, Festschrift 75 Jahre Eidgenössisches Versicherungsgericht, Berne 1992, p. 183-195, 183 ss.; Ulrich Meyer-Blaser, Die Zusammenarbeit von Richter und Arzt in der Sozialversicherung. Bull Méd Suisses. 1990;71:1090-5; le même, Sozialversicherungsrecht und Medizin, dans: Hermann Fredenhagen (éd.), Das ärztliche Gutachten, 4e édition, Berne etc. 2003, p. $21 \mathrm{ss}$
Cette définition légale est presqu'exclusivement tautologique selon l'énoncé «A $=A »$. La signifiance et donc la possibilité de réglementation normative sont par conséquent extrêmement restreintes. Cette constatation vaut au préalable pour le cœur de la notion:

\section{Maladie = toute atteinte à la santé physique,} mentale ou psychique

Quelque chose de négatif («maladie») est décrit comme la négativité («toute atteinte») de quelque chose de positif («la santé physique, mentale ou psychique»). La logique linguistique le traduit par la déclaration:

Maladie = toute maladie physique, mentale
ou psychique

\section{La fonctionnalisation de la notion de maladie}

Contrairement à une attente première, le caractère tautologique de la définition légale est également conservé lors de la prise en compte d'autres caractéristiques d'état de fait. L'être humain ne serait pas déjà malade au sens légal de l'assurance sociale, selon le libellé sur ce point clair de la loi, lorsqu'il est malade, mais seulement et quand, dans la mesure où, et tant qu'il nécessite à cause de cela des soins médicaux (examen et traitement [3]) ou (alternativement, la conjonction n'exprimant pas l'exclusion) qu'il est dans l'incapacité de travailler. Cette connexion de la notion de maladie, d'une part avec la nécessité de traitement [4], d'autre part avec l'incapacité de travailler, ne nous avance aucunement. Le malade qui supporte seul, en silence, son mal, qui n'est à charge de personne et fonctionne socialement, la maladie silencieuse qui incommode il est vrai son porteur mais pas tant qu'il ait besoin de l'aide de tiers, ne présentent d'emblée pas d'intérêt juridique. D'un point de vue du droit de l'assurance sociale, on n'est pas malade pour la maladie mais pour pouvoir faire valoir des prestations assurées: soins médicaux, indemnités ou rente d'invalidité. La relation entre la notion de maladie et l'incapacité de travailler est en particulier un cercle vicieux légal par excellence, l'incapacité de travailler se définissant comme étant une atteinte à la santé physique, mentale ou psychique, donc une incapacité due à la maladie d'accomplir une activité exigible (art. 6 LPGA). Le requérant aborde tout juste la question de ce qu'est la santé, la maladie, et la loi lui répond qu'il est malade quand il ne peut plus travailler suite à sa maladie.

\section{Résultat intermédiaire}

Le résultat à ce niveau de réflexion est, d'un point de vue médical, bland, négatif, nihil. Comme chaque définition juridique de la maladie, celle de l'art. 3 LPGA est correcte en elle-même; elle n'a toutefois pas de contenu originaire et de contours qu'une interprétation pourrait déterminer. Ainsi le droit est renvoyé à la médecine [6] .

\section{La maladie au sens médical}

Que signifie donc la maladie au sens médical? La réponse s'impose: la médecine ne le sait pas ellemême. L'histoire de la médecine montre bien plus le changement au fil des époques de la compréhension de la maladie, comme part de l'histoire de l'humanité marquée par des conceptions religieuses, philosophiques et des sciences physiques et naturelles se modifiant tout au long des décennies. Il existait, et il existe, quantités de concepts et de modèles médicaux: naturalisme ou normativisme, combinaisons avec ceux-ci, maladie comme anormalité, maladie dans un contexte social, modèle biomédical, modèle bio-psychosocial, approche systémique-psychosomatique, concept de la salutogenèse, modèle de Meikirch [7], et bien d'autres, laissent le droit sans boussole fiable [8]. Le diagnostic médical a indéniablement progressé avec la diffusion d'ICD-10 et DSM IV pour ce qui est de la fiabilité et de la constance (reliability). Cependant la question juridique, comment traiter les marges d'évaluation et d'appréciation, garde toute son actualitétant dans l'application des systèmes diagnostiques que dans la nécessaire détermination des restrictions de la capacité fonctionnelle de travailler et des ressources psychiques découlant des maux diagnostiqués. Il convient de différentier le bénin du grave.

\section{Situations individuelles d'application du droit}

\section{La maladie aiguë et la maladie grave}

La maladie aiguë, la déclaration d'une maladie comme elle se manifeste lors d'un infarctus du myocarde, d'une embolie, d'une crise de nerfs et autres revers de fortune, ne présentent pas de problème dans le cadre de l'art. 3 LPGA. Plus l'évolution d'une maladie s'aggrave - jusqu'à l'aveu d'impuissance médicale cité en avant-propos plus facilement les juristes admettront la notion de maladie. L'ordre de ce qui est et devrait être se trouve en totale conformité harmonieuse. En vérité, le mal grave au cours morbide et à l'issue fatale n'intéresse juridiquement pas. La maladie 
aiguë, notamment la maladie grave, l'est sans nul doute médicalement et l'est tout autant sans équivoque légalement.

\section{La douleur chronique}

Seules les maladies irréversibles ou qui durent longtemps, donc les maladies chroniques, sont importantes dans le droit sur l'invalidité pour les rentes en tant que prestations durables des différentes branches de l'assurance sociale. Ce ne sont pas les maladies chroniques graves qui présentent un intérêt juridique mais ici aussi ce ne sont que les évolutions d'états dont se plaignent incessamment les patients que même les médecins mettent, ou devraient mettre, en doute quant à leur valeur en tant que maladie. Au centre de la discussion interdisciplinaire se trouvent ainsi les domaines limites entre ce qui est sain et malade, entre la maladie lorsqu'elle est presqu'encore santé ou pourrait redevenir santé, lorsqu'elle est plus un comportement qu'un état. Ici se présente une large thématique à plusieurs niveaux ne pouvant être qu'abordée dans ce cadre.

Johannes Bircher, Macht Zumuoder gesund?, dans Erwin Murer (édit.), Was darf dem erkrankten oder verunfallten Menschen zu-gemutet werden? journées sociales fribourgeoises 2008, Berne 2008, p. 325 ss., 327 , note bas de page 4 avec de plus amples indications.

8 Pour tout le sujet voir Schwendener (note 2), p. 17 ss., 36 ss. Voir aussi Alexa Franke, Modelle von Gesundheit und Krankheit, Berne 2006, p. 117 ss., 157 ss.

9 Voir ATF 130 V 352, 130 V 396, 131 V 49, 132 V 65. Comp. conc. l'importance des facteurs psychosociaux et socioculturels, fondamental: ATF 127 V 294 et la récente littérature: Hans-Jakob Mosimann/Gerhard Ebner, «Objektiv nicht überwindbare» Erwerbsunfähigkeit: Überlegungen zu Art. 7 Abs. 2 ATSG aus juristischer und psychiatrischer Sicht, dans: Revue suisse des assurances sociales et de la prévoyance professionnelle (RSAS) 52/2008 p. 513 ss.; Jörg Jeger, Wer bemisst invaliditätsfremde (soziokulturelle und psychosoziale) Ursachen der Arbeitsunfähigkeit - der Arzt oder der Jurist?, dans: journée du droit des assurances sociales 2008 , IRP/HSG, tome 57 p. 147 ss.

10 Par exemple Jörg Jeger, Somatoforme Schmerzstörung und Arbeitsunfähigkeit: Differenzen oder Konsens zwischen Medizin und Rechtsprechung? dans: René Schaffhauser/Franz Schlauri (édit.), Medizin und Sozialversicherung im Gespräch, tome 35 de la série IRP/HSG, St. Gall 2006, p. 155-210, 191.

\section{L'incorporation du social}

\section{dans la notion médicale de la maladie}

La maladie de longue durée, chronique, a de par sa nature toujours existé et dès ses débuts, l'assurance sociale examinait les possibles conséquences d'une invalidité. Une différence essentielle dans la gestion de ce phénomène réside dans le fait que par le passé les représentants des disciplines médicales impliquées, notamment l'orthopédie et la rhumatologie, décrivaient la maladie chronique de façon plus nette comme étant une entité médicale relativement bien délimitable.

Avec l'entrée d'ICD-10 en médecine, en application de la définition de l'OMS de la santé comme bien-être physique, psychique et social intacts, des états de maladie furent diagnostiqués dans le cadre desquels la situation sociale joue un rôle déterminant pour leur cause et leur maintien.

\section{La réalité subjective, raison suffisante pour reconnaître médicalement l'état de maladie}

Le traitement médical (mis à part d'autres buts, par exemple à caractère sanitaire, ne faisant pas ici l'objet d'une discussion) est dédié au bien-être de la patiente et du patient. La subjectivité de la souffrance et de l'expérience de la maladie est une réalité pour la personne touchée que le médecin, thérapeute, ne peut mettre de côté. Celui qui, subjectivement, se sent malade, est médicalement malade. Celui qui se croit incapable de travailler doit véritablement l'être, vu sous l'angle contraignant de la subjectivité du patient.

\section{Voies de sorties du dilemme}

La sociogenèse et la reconnaissance médicale d'états maladifs subjectifs en tant que maladies, parfois renforcées par le fait que les implications de l'obligation d'assurance et de la gestion de la maladie sont occultées, voire consciemment niées, confrontent l'ordre juridique à des problèmes conséquents. Cela vaut tout particulièrement pour le droit de l'assurance sociale qui doit décider qui a droit, à quelles conditions, à des prestations à charge de la communauté des assurés. Il n'existe pas un domaine de l'ordre juridique où des états internes indémontrables et où l'autoévaluation subjective de la personne ne soient reconnus à eux seuls comme bases fondant un droit et protégés légalement.

Des états internes indémontrables et l'autoévaluation subjective de la personne assurée ne constituent juridiquement pas à eux seuls une base de droit.

En clair cela ne signifie pas, pour éviter un double malentendu, que seules des maladies médicalement explicables donnent droit à une rente, non plus que de telles maladies donnent toujours droit à une rente. Il est toutefois inévitable que le droit examine de manière critique notamment la valeur de la maladie et les effets d'états étiologiques-pathogénétiques vagues d'atteinte à la santé sur la capacité de travailler quand la perception et l'appréciation propres, les facteurs psychosociaux ou socioculturels sont à l'avant-plan. Reste à savoir à quel niveau cela doit se faire.

\section{Pas par le biais de la notion de maladie}

La correction normative par la jurisprudence [9], critiquée par le domaine médical [10], ne s'est pas faite au niveau de la notion de maladie. L'application juridique de l'assurance-maladie selon l'art. 3 al. 1 LPGA le montre bien. Il est juridiquement indiscutable qu'une personne souffrante reçoive les traitements médicaux nécessaires et que ces mesures soient en principe à charge de l'assurance-maladie. Une dichotomie de la notion de maladie bio-psycho-sociale dans la médecine, du modèle biopsychique dans l'assurance sociale doit être rejetée afin de garder l'ordre de ce qui est et devrait être. Le droit ne peut se fermer aux développements que la médecine a connus ces dernières années et qui se poursuivent. Il serait présomptueux de vouloir supposer que le droit de l'assurance sociale pourrait inverser la vapeur et prescrire à la médecine l'application d'une notion de maladie qui ne correspond plus depuis longtemps à l'état des connaissances scientifiques. Du reste, par l'approbation de la notion 
11 Comparer avec la note 10.

12 Par exemple Hardy Landolt, Das Zumutbarkeitsprinzip im schweizerischen Sozialversicherungsrecht, unter besonderer Berücksichtigung der Rechtsprechung des Eidgenössischen Versicherungsgerichts, Zurich 1995 p. 269 s.; Thomas Locher, Die invaliditätsfremden Faktoren in der rechtlichen Anerkennung von Arbeitsunfähigkeit und Invalidität, dans: René Schaffhauser/ Franz Schlauri (édit.), Schmerz und Arbeitsunfähigkeit, tome 23 de la série IPR/HSG, St. Gall 2003 , p. 243-258, 254 s.; Hans-Jakob Mosimann, Somatoforme Störungen: Gerichte und (psychiatrische) Gutachten, dans: RSAS 43 (1999), p. 1-21 ( $1^{\text {re }}$ partie), p. $105-128$ ( $2^{\mathrm{e}}$ partie), p. 15 ss., 114 ss.; Erwin Murer, die rechtlichen Anforderungen an sozialmedizinische Begutachtungen im Bereich zwischen Psyche und Soma, dans: Gerhard Ebner/Volker Dittmann/ Bruno Gravier/Klaus Hoffmann/ René Raggenbass (édit.), Psychiatrie und Recht/Psychiatrie et Droit (forum gesundheitsrecht/droit de la santé, tome10), Zurich/Bâle/ Genève 2005, p. 91-124, 102 ss.; le même, Die UV- und IV-rechtliche Auseinandersetzung mit psychischen Störungen - eine Zwischenbilanz, dans: Erwin Murer (édit.), Psychische Störungen und die Sozialversicherung Schwerpunkt Unfallversicherung journée de droit social fribourgeoise 2002, Berne 2002, p. 1-56, $41 \mathrm{~s}$.

13 ATF 105 V 156 consid. 1 p. 158 et la jurisprudence constante depuis

14 Ulrich Meyer, Die Beweisführung im Sozialversicherungsrecht, dans: Erwin Murer (édit.), Nicht objektivierbare Gesundheitsbeeinträchtigungen: Ein Grundproblem des öffentlichen und privaten Versicherungsrechts sowie des Haftpflichtrechts, journées de droit social fribourgeoises 2006, Berne 2006, p. 199-225, 217 ss.

15 Dans ce sens, l'arrêt I 70/07 du 14 avril 2008. de maladie dans le cas particulier, seule la première marche du système de prestations légal est gravie; quant aux autres obstacles que constituent la capacité de travailler, l'accomplissement d'une activité lucrative et l'invalidité, rien n'est décidé car il reste aux experts médicaux la tâche de décrire les effets des troubles diagnostiqués sur les fonctions et les capacités de la personne requérant une rente d'invalidité.

\section{Par l'exigibilité?}

La jurisprudence [11], avec elle la plus grande partie de la doctrine [12], a parcouru ce chemin. Juridiquement il repose sur l'art. 6 LPGA qui se rapporte à la capacité existante d'accomplir un travail exigible. Mis à part cela, l'exigibilité correspond à un principe général de droit qui, comme le devoir de réduire le dommage, est utilisé même sans base légale. L'appréciation de l'exigibilité ne règle toutefois pas la contradiction entre la médecine et le droit. Il faut faire comprendre à la personne touchée qu'elle est, d'un point de vue médical, certes malade et incapable de travailler mais que juridiquement elle ne le sera pas car les critères de morbidité déontiques en jurisprudence pour donner droit à une prestation ne sont pas remplis. Ici réside aussi pour l'expert médical une situation normative initiale difficile à surmonter car sa tâche se limite à juger de l'état de santé (observations, diagnoses) et à prendre position quant à l'incapacité de travailler (décrire les restrictions, dues à l'état de santé, de la capacité de fonctionner et des ressources psychiques); de plus ses indications sont une base importante dans l'appréciation juridique des prestations de travail exigibles. [13]

\section{Par la preuve ...}

Même le médecin, qu'il soit médecin traitant ou expert, ne saurait s'offusquer d'un refus si la base pour faire valoir le droit n'est pas prouvée ou ne peut l'être. Cet empêchement de produire la preuve se rencontre par nature souvent dans les caractéristiques indéfinies d'incommodités étiologiques-pathogénétiques. Cette pensée doit être reliée aux règles de preuve reconnues appliquées dans l'organisation judiciaire de l'assurance sociale [14]. Si une caractéristique avec ses conséquences sur la capacité de travailler - malgré des éclaircissements soigneusement menés - reste floue, indéterminée, la preuve pour faire valoir un droit n'est pas apportée et ne peut l'être. Le principe est donc le suivant: des états médicaux aux syndromes indéterminés sans caractéristiques saisissables ne peuvent fonder un droit à des prestations d'invalidité à durée indéterminée.

\section{... en relation avec une attribution différentiée de prestations}

Malgré cela le problème n'est pas réglé. La large propagation - du moins perçue ainsi par la dernière instance - on serait presque tenté de dire la propagation épidémique, de douleurs chroniques, de peur, d'insomnies, de vertiges, de faiblesses, d'épuisement, de manque d'entrain, de fatigue chronique, de burn out, d'indispositions dues à l'environnement, et bien d'autres, reflète les maux de notre temps; les temps sont durs, les exigences professionnelles nettement supérieures à celles des années 80 . Ces maux sociaux sont aujourd'hui médicalement reconnus. Ne seraitil pas conséquent de les reconnaître intégralement dans l'assurance sociale et de les accepter comme base pour accorder des rentes d'invalidité? Après ce qui a été dit, la réponse ne peut être que négative car cette conséquence n'est pas voulue par le législateur - selon toutes les sources de connaissances à disposition - et les systèmes légaux de prestations - financés par les cotisations et les im-pôts - ne sont pas adaptés pour cela. Il faudrait bien plus réfléchir à une indemnisation différentiée, limitée, de tels états de maladie diffus, en fait à une indemnité - instrument légal prévu à cet effet - ou, là où elle n'existe pas, comme dans l'assurance-invalidité, à une rente limitée dans le temps pour autant que l'irruption de la maladie et son déroulement le justifient [15]. 